\title{
Interactive comment on "The silica-carbon biogeochemical cycle in the Bohai Sea and its responses to the changing terrestrial loadings" by Jun Liu et al.
}

Anonymous Referee \#1

Received and published: 21 March 2016

Please see the attached pdf file.

Please also note the supplement to this comment:

http://www.biogeosciences-discuss.net/bg-2016-42/bg-2016-42-RC1-supplement.pdf

Interactive comment on Biogeosciences Discuss., doi:10.5194/bg-2016-42, 2016. 